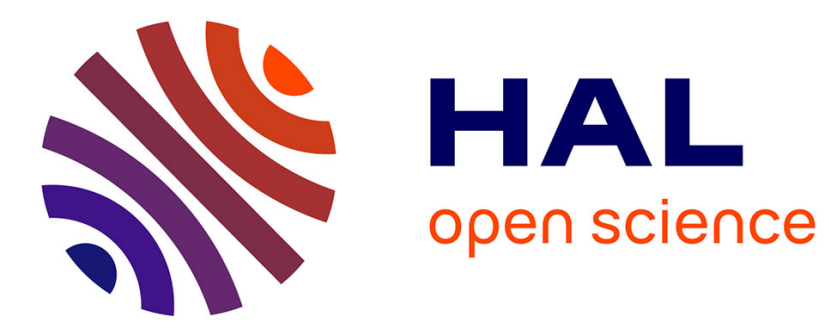

\title{
Photo-assisted recovery in ammonia sensor based on organic vertical diode
}

Yu-Ting Lin, Shang-Yu Yu, Hsiao-Wen Zan, Ping-Hung Yeh, Chia-Jung Lu, Hsin-Fei Meng, Chih-Wei Luo, Olivier Soppera

\section{- To cite this version:}

Yu-Ting Lin, Shang-Yu Yu, Hsiao-Wen Zan, Ping-Hung Yeh, Chia-Jung Lu, et al.. Photo-assisted recovery in ammonia sensor based on organic vertical diode. Organic Electronics, 2019, 67, pp.272-278. 10.1016/j.orgel.2019.01.036 . hal-02323194

\section{HAL Id: hal-02323194 \\ https://hal.science/hal-02323194}

Submitted on 25 Nov 2020

HAL is a multi-disciplinary open access archive for the deposit and dissemination of scientific research documents, whether they are published or not. The documents may come from teaching and research institutions in France or abroad, or from public or private research centers.
L'archive ouverte pluridisciplinaire HAL, est destinée au dépôt et à la diffusion de documents scientifiques de niveau recherche, publiés ou non, émanant des établissements d'enseignement et de recherche français ou étrangers, des laboratoires publics ou privés. 


\title{
Photo-Assisted Recovery in Ammonia Sensor Based on Organic
}

\section{Vertical Diode}

Yu-Ting Lin ${ }^{a}$, Shang-Yu Yu ${ }^{\text {b,c }}$, Hsiao-Wen Zan ${ }^{\text {** }}$, Ping-Hung Yeh ${ }^{\mathrm{d}}$, Chia-Jung Lu ${ }^{\mathrm{e}}$, Hsin-Fei Meng ${ }^{\mathrm{f}}$, Chih-Wei Luo ${ }^{\mathrm{a}}$, and Olivier Soppera ${ }^{\mathrm{c}}$

${ }^{a}$ Department of Electrophysics, ${ }^{b}$ Department of Photonics , College of Electrical and Computer Engineering, and ${ }^{\mathrm{f}}$ Institute of Physics, National Chiao Tung University, No.1001, University Rd., East Dist., Hsinchu City 300, Taiwan

c Université de Haute-Alsace, CNRS, IS2M UMR 7361, F-68100 Mulhouse, Université de Strasbourg, France

${ }^{\mathrm{d}}$ Department of Physics, Tamkang University, No.151, Yingzhuan Rd., Tamsui Dist., New Taipei City 25137, Taiwan

${ }^{\mathrm{e}}$ Department of Chemistry, National Taiwan Normal University, No.162, Heping East Rd., Section 1, Taipei City 106, Taiwan

*Corresponding authors

E-mail :

hsiaowen@mail.nctu.edu.tw (Hsiao-Wen Zan)

phyeh331@mail.tku.edu.tw (Ping-Hung Yeh)

\begin{abstract}
In this article, a photo-assisted gas sensor was provided to solve the slow recovering issue of the organic gas sensor. The proposed organic gas sensor can detect ammonia in parts-per-billion regime in 30 seconds and can be multiple uses, but the long recovery time about 5 mins places difficulty to realize rapidly multiple-time sensing. Therefore, we used the photo-absorption ability in the organic semiconductor layer to generate the photocurrent and hence to compensate the current drop due to the ammonia absorption. The recovery time was significantly reduced to be only $1 \mathrm{~min}$. The specific wavelength was also found to match the absorption spectrum in different organic sensing materials. As a comparison, the quartz crystal microbalance (QCM) was used to clarify the gas absorption and desorption mechanism. It is believed that this photo-assisted gas detection could be a general method to solve the slow recovery in organic-based gas sensors.
\end{abstract}

\section{Keywords}

Ammonia, fast recovery, light-assisted recovery, light emitting diode, organic gas sensor 


\section{Graphical Abstract}

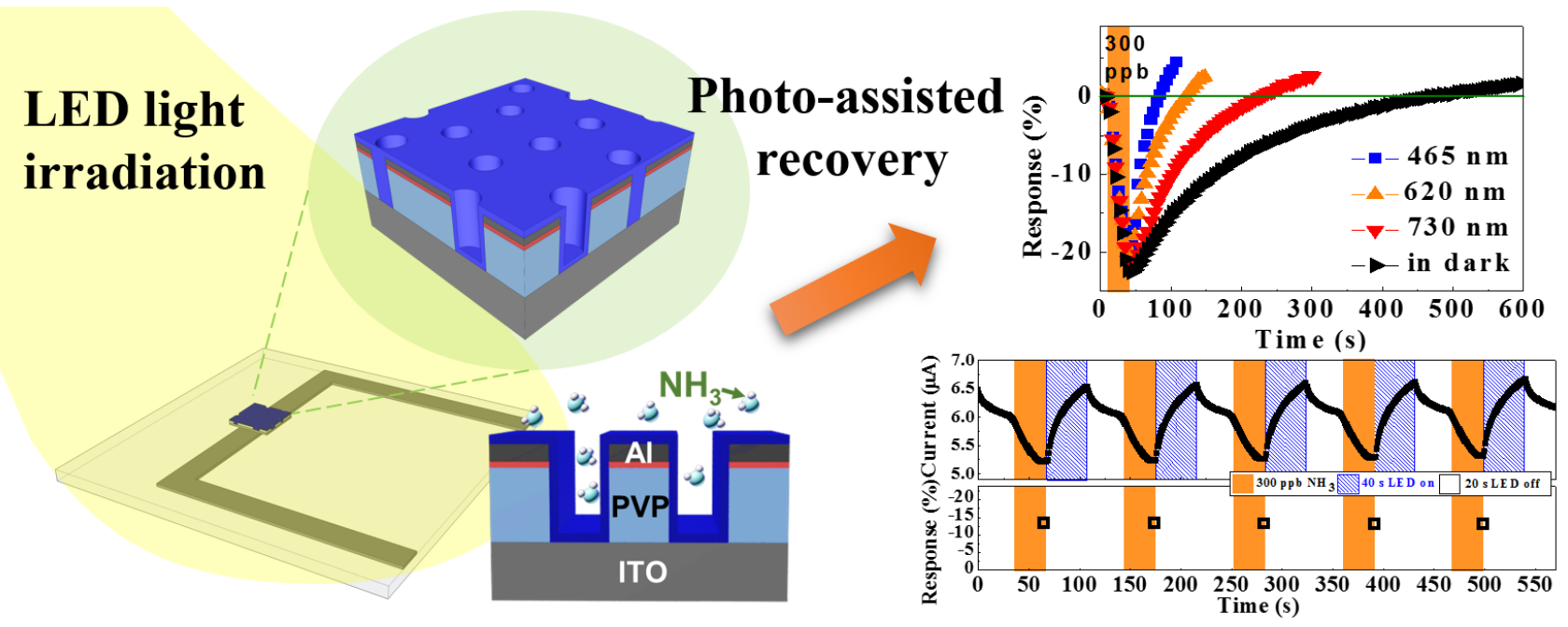

\section{Introduction}

With the emerging development of the internet of things (IoT) technologies, the need for various kinds of solid-state sensors appears to be more and more important. Notably, the sensors for detecting the human body signals to reflect the health conditions are of high demand. In recent years, organic semiconducting devices are reported to exhibit a very high sensitivity to ammonia gas molecules [14]. Traditionally, ammonia detection is used in ambient air quality monitoring which requires the detection range in parts-per-million ( $\mathrm{ppm}$ ) regime. Recently, the ammonia detection in parts-per-billion $(\mathrm{ppb})$ regime was reported to be useful in monitoring the spoilage of fish or several kinds of aquaculture products [5,6], in controlling the clean room ambient, and in detecting ammonia in breath to reflect the health conditions of patients with chronic kidney diseases [7-9] or with Helicobacter pylori infection [10]. Organic semiconductor devices exhibit the advantages of low production cost and the ability to detect ammonia in ppb-regime. Thus, they are promising to be used to develop abovementioned applications.

However, to push into the real applications, the requirements on sensors are more than high sensitivity and low production cost. Key factors such as selectivity, a long lifetime in the air, and multiply-used ability are all critical. In prior reports, organic-based ammonia sensors can be roughly divided into two kinds of devices. The first kind uses a transistor structure [11-13]. The second kind uses the vertical diode structure which was proposed by our group [7, 14, 15]. For both transistor structure and vertical diode structure, the sensors have ppb-regime sensitivity to ammonia. The selectivity among ethanol, acetone, nitric oxide, carbon dioxide is also verified to be good enough in prior works [16-18]. The long-enough lifetime in air (64 days) was reported by our prior work [7]. The multiple-used stability in air was also verified by many groups [19-22]. However, all the reported works suffer from a slow recovery time (i.e., 3-10 mins), compared to the fast detecting time (i.e., 5$60 \mathrm{~s}$ ). The slow recovery time places a limitation on the applications. For example, for in-line meat 
freshness detection or for breath ammonia detection in the hospital, the 5 mins recovery causes user impatient and a long queue of waited samples.

Here in this work, we fabricated a sensitive ammonia sensor by using an air-stable donor-acceptor (D-A) type polymer, Poly[[4,8-bis[5-(2-ethylhexyl)-2-thienyl]benzo[1,2-b:4,5-b']dithiophene-2,6diyl][2-(2-ethyl-1-oxohexyl)thieno[3,4-b]thiophenediyl]] (PBDTTT-C-T). Then, we proposed a lightassisted method to greatly reduce the recovery time from $300 \mathrm{~s}$ to $60 \mathrm{~s}$. The wavelength dependency on the photo-assisted recovery time was observed. Also, the multiple sensing stability is confirmed to be good enough with the light-assisted recovery. The mechanism is also investigated by comparing the light-induced current variation in the organic vertical diode and the light-induced desorption in quartz crystal microbalance (QCM) devices. We concluded that the light-assisted recovery is mostly achieved by compensating the effect of ammonia-induced carrier reduction with the light-generated photocarriers.

\section{Experimental section}

\subsection{Sensor fabrication}

Fig. 1(a) shows the fabrication process of the ammonia sensor with vertical channels on the sidewall of cylindrical nanopores [7]. The cylindrical nanopores were fabricated via the colloidal lithography method. Firstly, a glass substrate with patterned indium tin oxide (ITO) (sheet resistance as $6 \mathrm{Ohm} /$ square) was treated with oxygen plasma $(100 \mathrm{~W} 600 \mathrm{~s})$ to form a hydrophilic surface. A cross-linkable Poly(4-vinylphenol) (PVP) layer (thickness $\sim 300 \mathrm{~nm}$ ) was then spin-coated on the ITO substrate and annealed at $200{ }^{\circ} \mathrm{C}$ for $1 \mathrm{hr}$. to serve as the insulating layer. To modify the PVP surface to favor the subsequent adhesion of negatively charged polystyrene (PS) nano-spheres (Fluka, $200 \mathrm{~nm}$ diameter), a thin layer of Poly(3-hexylthiophene-2,5-diyl) (P3HT) was spin-coated onto PVP and annealed at $200{ }^{\circ} \mathrm{C}$ for 10 mins. Then, the substrate was immersed into PS solution ( $10 \mathrm{wt} \%$ in ethanol) for $90 \mathrm{~s}$ and dipped into boiling isopropanol (IPA) for $10 \mathrm{~s}$ to remove excess PS spheres. After quickly drying out the substrate with nitrogen flow, the monolayer PS nano-spheres was formed to serve as a shadow mask for Al electrode deposition. After evaporating a 60-nm-thick Al layer (evaporation rate as $0.15 \mathrm{~nm} / \mathrm{s}$ ), the PS nano-spheres were then peeled off with 3M Scotch Tape to obtain porous Al top electrode. The porous top electrode was further served as another shadow mask for subsequent oxygen plasma etching. During the plasma etching process, the PVP in the area not covered by the top electrode was removed, thus forming the cylindrical nanopore structure. Finally, two different types of active layer were deposited onto the structure and annealed at different temperatures, enabling the conduction of vertical current. For the proposed air-stable device, PBDTTT-C-T ( $0.9 \mathrm{wt} \%$ in chlorobenzene) was blade coated onto the nanopore substrate with the blade speed of $200 \mathrm{~mm} / \mathrm{s}$. The substrate was, then, annealed at $80{ }^{\circ} \mathrm{C}$ for 10 mins. The resulting thickness of PBDTTT-C-T is around $40-50 \mathrm{~nm}$. For the control sample, P3HT (1.5 $\mathrm{wt} \%$ in chlorobenzene) was spin-coated on to the substrate and was annealed at $200{ }^{\circ} \mathrm{C}$ for 10 mins. The thickness of P3HT is around $35-45 \mathrm{~nm}$. 


\subsection{Sensing system with a light irradiation control}

The gas sensing system is as depicted in Fig. S1 in supporting information. The system comprised a transparent sensing chamber, a desiccation cylinder (sodium hydroxide $(\mathrm{NaOH})$ ) to provide a steady relative humidity $(\mathrm{RH})$ around $10 \%$ and eliminate the interference from ambiance $\mathrm{RH}$ variation, an electric source measurement unit (Keysight U2722A USB modular) to apply operational voltage on sensor and read out electric signal, a rotameter to control the flow at $500 \mathrm{cc} / \mathrm{min}$, a pump to provide a constant air flow, and a humidity measurement unit to confirm the RH value during experiment. To study the effect of light irradiation and to prevent the light interference from the ambiance, the transparent sensing chamber with the mounted sensor was placed inside an opaque box, as shown in Fig. 1(b). Different light-emitting diodes (LEDs) were utilized to irradiate light onto the sensor, sequentially. The wavelength and the irradiance of various LED light sources are $465 \mathrm{~nm}$ (630 $\left.\mu \mathrm{W} / \mathrm{cm}^{2}\right), 620 \mathrm{~nm}\left(210 \mu \mathrm{W} / \mathrm{cm}^{2}\right)$, and $730 \mathrm{~nm}\left(12 \mu \mathrm{W} / \mathrm{cm}^{2}\right)$.

\subsection{QCM measurement}

To investigate the molecular absorption/desorption during light irradiation, we formed the identical sensing layer, PBDTTT-C-T with 40-50 nm thickness, onto the QCM chip (purchased from Seiko) by using the dip-coating process. Then, the frequency of QCM chip was measured by using QCM922A (Seiko) upon ammonia injection/removal when the QCM chip was placed in dark or under light irradiation.

\section{Results and discussion}

The scanning electron microscope (SEM) image of the nanoporous substrate was shown in Fig. 2(a). This nanoporous structure (or 3D structure) increases the detection area, and the vertical channel greatly reduces the channel length to be $300 \mathrm{~nm}$. Hence, the sensor exhibits a high sensitivity to low concentration ammonia (i.e., $100 \mathrm{ppb}$ ) and delivers high output current as a few microamperes at $5 \mathrm{~V}$. It is noted that organic semiconductor material usually exhibits low mobility, the hole mobility for PTBTTT-C-T was $1.3 \times 10^{-2} \mathrm{~cm}^{2} \mathrm{~V}^{-1} \mathrm{~s}^{-1}$ in the prior report [23]. The current-voltage characteristic of the PBDTTT-C-T sensor was shown in Fig. 2(b). It could be observed that at the turn-on voltage around $4 \mathrm{~V}$, the turn-on curve shows a Schottky conduction behavior, which is due to the presence of injection barrier from ITO (work function as $5 \mathrm{eV}$ after oxygen plasma treatment) to PBDTTT-C-T (highest occupied molecular orbital, HOMO, as $5.2 \mathrm{eV}$ ). The real-time sensing phenomenon was shown in Fig. 2 (c) by plotting sensing responses as a function of time. The orange background region indicates the periods when the ammonia with different concentrations (100 ppb to $1 \mathrm{ppm})$ was injected into the sensing chamber. The carrier gas is dry air with relative humidity around $10 \%$, and the pump rate is fixed as $500 \mathrm{cc} / \mathrm{min}$. The sensing response is calculated by the current variation value divided by the initial current value right before gas injection. The sensing period was set as $30 \mathrm{~s}$. It could be seen that 
with only $30 \mathrm{~s}$ ammonia injection, the sensor exhibits substantial responses to different concentration of ammonia. Specifically, the sensing response to $100 \mathrm{ppb}$ ammonia reaches $14.9 \%$. Even though the sensing requires only $30 \mathrm{~s}$, in Fig. 2(c), we noticed that the current recovery is rather slow. After 100 ppb ammonia detection, it takes $183 \mathrm{~s}$ of recovery time for the current returns to its original current level, denoting the zero response value. With increasing ammonia concentration, the recovery time is significantly increased from $183 \mathrm{~s}$ to $490 \mathrm{~s}$ for $1000 \mathrm{ppb}$ ammonia detection. However, the sufficient recovery time is of paramount requirement for multiple sensing performances in the organic sensor. If the multiple testing is done with insufficient recovery time, the response becomes smaller and smaller along with the testing times. As shown in Fig. 2(d), for the repeated $300 \mathrm{ppb}$ ammonia testing for five times, the normalized responses stayed almost unchanged when the recovery time is $300 \mathrm{~s}$ while the normalized response gradually decreases when the recovery time is $180 \mathrm{~s}$ (insufficient recovery). If the ammonia concentration increases to be $2 \mathrm{ppm}$ and the recovery time is only $180 \mathrm{~s}$ (insufficient recovery), then the normalized response significantly drops along with test times. Hence, sufficient recovery is needed to keep the sensor stability. However, such a long recovery time then places a limit on the rapidly multiple-time testing applications.

To reduce the recovery time, we may think of enhancing the gas desorption by increasing the pumping flow rate or by heating up the samples. However, the two approaches also take time to reach a stable condition. So, they are not suitable if the targeting recovery time is within 1 minute. In this study, we propose a light irradiating method to reduce the recovery time (photo-assisted recovery). The set-up is shown in Fig. 1(b). To study the influence of light irradiating on recovery phenomenon and its dependency on light wavelength, the light recovery performance of two different sensors, PBDTTT-C-T sensor and P3HT sensor, was investigated. The P3HT sensor was chosen as a control sample to study the wavelength dependency on the photo-assisted recovery due to its absorption wavelength different from that of PBDTTT-C-T. The absorption spectrums of these two materials were shown in Fig. 3(a). The P3HT exhibited an absorption peak around $515 \mathrm{~nm}$, whereas those of PDBTTT$\mathrm{C}$-T were at $645 \mathrm{~nm}$ and $704 \mathrm{~nm}$. The response as a function of time for PBDTTT-C-T sensor and P3HT sensor were plotted in Figs. 3(b) and 3(c), respectively. The orange regions indicate the $30 \mathrm{~s}$ injection of $300 \mathrm{ppb}$ ammonia. After a $30 \mathrm{~s}$ sensing time, the LED is turned on to irradiate the sample. Total 3 LEDs with the wavelength of $730 \mathrm{~nm}, 620 \mathrm{~nm}$, and $465 \mathrm{~nm}$ were used in this study. The irradiance of each LED was $12 \mu \mathrm{W} / \mathrm{cm}^{2}, 210 \mu \mathrm{W} / \mathrm{cm}^{2}$, and $630 \mu \mathrm{W} / \mathrm{cm}^{2}$, respectively. The response curves with different LEDs irradiating and without LED irradiating (i.e., in dark) are compared. In Fig. 3(b), the real-time response curve of PBDTTT-C-T sensor, we can observe that compared to the recovery curve obtained in dark, the recovery process was faster when the sensor was irradiated with LED light, and the recovery time of the sensor irradiated with different LED light source were varied. Moreover, the same phenomenon can be observed in P3HT sensor as shown in Fig. 3(c). However, for P3HT sensor, the recovery curve obtained by $730 \mathrm{~nm}$ LED irradiation showed no significant different to that of in dark, indicating that $730 \mathrm{~nm}$ light has no significant effect on the recovery process. The results above revealed that the effect of photo-assisted recovery is linked to two factors, the absorbance 
of the sensing material and the irradiance of the LED light source. To understand the relationship of these two factors to the photo-assisted recovery process, we, therefore, extracted the absorbance intensity of both PBDTTT-C-T and P3HT materials at the center wavelength of 3 LEDs (456nm, 620 $\mathrm{nm}$, and $730 \mathrm{~nm}$ ) and the recovery time of both sensors under the irradiation of 3 LEDs, and plotted as a function of LED light source as shown in Fig.3 (d). It can be observed that with the LED light source of $730 \mathrm{~nm}$, the PBDTTT-C-T exhibited a greater absorption than that of P3HT which is nearly negligible. Hence, with the relatively low irradiance of $730 \mathrm{~nm}$ LED irradiation, the PBDTTT-C-T sensor showed a huge reduction of recovery time, whereas no significant effect was found in the P3HT sensor. At the wavelength of $620 \mathrm{~nm}$, an alike absorbance can be seen in both material, resulted in a similar effect of the reduction of recovery time. Nevertheless, with the $465 \mathrm{~nm}$ LED irradiation, the P3HT sensor displayed a huge reduction of recovery time compared to that of PBDTTT-C-T sensor due to the great absorption property of P3HT material to $465 \mathrm{~nm}$ light source. Moreover, thanks to the strong irradiance of $465 \mathrm{~nm}$ light source, the recovery time of the PBDTTT-C-T and P3HT sensors can be reduced to $40 \mathrm{~s}$ and $2 \mathrm{~s}$, respectively. Finally, since our targeting recovery time is within 1 minute and the maximum irradiance of LEDs were limited by the output performance of LEDs, we choose $465 \mathrm{~nm}$ LED as the light source in the following experiments. Also, even though P3HT has a smaller recovery time, P3HT sensors has only 2-days lifetime in air (Fig. S2 in the supporting information). On the contrary, PBDTTT-C-T sensor exhibits a more than 15-days lifetime. Hence, in the following section, we choose PBDTTT-C-T as the proposed gas sensor and use $465 \mathrm{~nm}$ LED to study the mechanism of light/sensor interaction.

As previously discussed, sensor current can be quickly recovered with the $465 \mathrm{~nm}$ LED irradiating. However, to be ready for the next testing, LED needs to be turned off to keep the sensor in dark again. It is noted that, if the sensor is always operated under LED irradiation, the sensing response will degrade due to the constant elevation of the background carrier density under irradiation. Hence, after turning off the LED, an additional time is needed to stabilize the sensor current because the light current generated by LED is removed from the sensor. As a result, for every gas sensing test, the operating sequence was designed as the following three steps (in Fig. 4(a)). (I) Injecting ammonia for 30 s. (sensing period). (I) Irradiating LED for 40 s. (recovery period). (III) Turning off LED and stabilizing sensor current for $20 \mathrm{~s}$. (stabilizing period). It is noted that the current drops in stabilizing period because the photo-generated carriers are reduced after turning off the LED. The sufficient duration for recovery period and for stabilizing period can be confirmed by plotting the current variation as a function of time in Fig. 4(b). When turning on the LED, the current increases suddenly, hence a positive current variation is obtained. Then, during the $30 \mathrm{~s}$ of the irradiation, the current gradually increases to reach a saturated value and the current variation reduces to be almost zero. Therefore, it is enough to set the $40 \mathrm{~s}$ light irradiation as the "recovery period" to reach a full recovery. When turning off the LED, the current drops and the current variation is negative. After $20 \mathrm{~s}$, the current variation becomes almost zero. As a result, we use $20 \mathrm{~s}$ to define the stabilizing period.

With the proposed operation sequence, we then verify the feasibility by performing multiple 
testing. We firstly examined if the sensing response can be kept during 5-times $300 \mathrm{ppb}$ ammonia sensing in Fig. 4(c). The sensing periods (30 s ammonia injection), the recovery periods (40 s LED on), and the stabilizing periods ( $20 \mathrm{~s}$ ) are represented by orange, blue, and white regions, respectively. With the fast photo-assisted recovery, the multiple testing can be done within $90 \mathrm{~s}$. The responses for each time of sensing were plotted in the lower part of the figure. A good enough sensing stability can be observed. We further verify the multiple-time sensing performance by injecting ammonia with various concentrations for 20 times. As shown in Fig. 4(d), the ammonia concentration as $100 / 300 / 500 / 1000 / 2000 \mathrm{ppb}$ is injected into the sensing chamber in a random order. The final responses are plotted as a function of concentration in Fig. 4(e). For every different concentration, at least 3-times testing are done. The small standard deviations in Fig. 4(e) verify that the proposed photoassisted recovery method is suitable to realize a fast and stable multiple-times ammonia sensing. It is noted that the response in continuously multiple testing is smaller than the single time testing. For example, when detecting $300 \mathrm{ppb}$ ammonia for one time, the response is around $23 \%$ in Fig. 2(c). In photo-assisted multiple testing mode, the response reduces to be about $13 \%$ as in Fig. 4(a). The phenomena make us suggest that the photo-assisted fast recovery is not due to the enhancement of the ammonia desorption, but a superposition of the current increase under light irradiation onto the current drop under gas sensing. Hence, with the remaining ammonia inside the active layer, the response becomes smaller in the multiple testing mode. However, as shown in Fig. 4(d), the response reaches a new stabilized condition and successfully delivers a stable calibration curve in Fig. 4(e).

Then, we like to investigate the underlying mechanism of the photo-assisted recovery. The primary interest is to clarify if the there is any enhanced gas desorption under light irradiation. In volatile organic compounds (VOCs) sensors based on oxide material such as ZnO, UV light is usually used to enhance the density of the weak-bounded oxygen and then to improve the reaction between VOC species and the absorbed oxygen [24]. Here, in ammonia sensor based on the organic semiconductor material, it is believed that the current reduction in the p-type organic semiconductor is because that the electron lone pair in ammonia molecules serve as traps for holes to de-dope the semiconductor and hence reduce the current $[7,25,26]$. Under light irradiation, additional photogenerated carriers increase the current of the device. Hence, it is possible that the photo-assisted recovery is simply the superposition of the gas sensing current plus the light detecting current. To verify the proposed mechanism, we designed two sets of experiments. In the first experiment, we measure the pure gas sensing response and the pure light irradiating response as shown in Fig. 5. The response is the current variation ratio. The black curve represents the response under $30 \mathrm{~s}$ detection $(\mathrm{t}$ $=0 \mathrm{~s} \sim \mathrm{t}=30 \mathrm{~s}$ ) of $300 \mathrm{ppb}$ ammonia and then recovered in dark. The blue curve represents the response of the sensor without contacting any ammonia and was irradiated by light at $t=30 \mathrm{~s}$. When adding the black and the blue lines together, the result is the green line. Finally, the response of the sample exposed to ammonia and then irradiated by LED is also plotted in Fig. 5 as the red line. A good overlap between the green and the red lines is obtained, indicating that the photo-assisted recovery is simply using the photo-generated carrier to overcome the current drop during the ammonia sensing. There may be no 
enhanced gas desorption during light irradiation. To further confirm this phenomenon, we did the second set of experiment. We utilized the quartz crystal microbalance (QCM) to help examine if there is any enhanced ammonia desorption from PBDTTT-C-T during LED irradiation. The result is shown in Fig. 6(a), in which the frequency shift of the PBDTTT-C-T coated QCM chip is plotted as a function of time. The orange region indicates the $30 \mathrm{~s}$ injection of $300 \mathrm{ppb}$ ammonia, then the recovery is observed in dark and under LED irradiation. In the recovery period, in the initial $30 \mathrm{~s}$, LED irradiation indeed enhance the desorption of ammonia, so the frequency shift exhibit a fast recovery in the beginning $30 \mathrm{~s}$. However, after $\mathrm{t}=60 \mathrm{~s}$, the QCM sample has a prolonged recovery. The full recovery is achieved after $273 \mathrm{~s}$ for the sample under LED irradiation. When we plot the PBDTTT-C-T sensor response as a function of time in Fig. 6(b), the orange region also represents the $30 \mathrm{~s}$ injection of 300 ppb ammonia. After removing the ammonia, the response quickly recovers to be zero within $30 \mathrm{~s}$. We hence conclude that, under LED irradiation, there is a little bit light enhanced desorption. However, the main reason to achieve a fast recovery is because the light generated current compensates the current reduction due to ammonia. The proposed mechanism is illustrated in Fig. 6(c). When PBDTTTC-T sensor is exposed to ammonia, the absorbed ammonia molecules serve as hole trapping centers to reduce the hole current. Then, after turn on LED light, the photo-generated current provides additional electron-hole pairs to increase the current level. The ammonia molecules still undergo a slow desorption process. The successful multiple testing in Fig. 4 verifies that, even with remaining ammonia molecules, the PBDTTT-C-T sensor can repeatedly response to the absorbed ammonia in the multiple tests. It is believed that the multiple testing is successful because the ammonia concentration in this study is low. The reacting-site density in PBDTTT-C-T is much higher than the absorbed ammonia density. Hence, the sensor current can keep detecting newly absorbed ammonia molecules even when there are remaining ammonia molecules inside the sensing film.

\section{Conclusions}

In this work, we provided a photo-assisted gas detection method to improve the recovery time and solve the dilemma. The PBDTTT-C-T organic semiconductor was chosen as the sensing material to provide good sensitivity to ammonia in parts-per-billion (ppb) regime and also to exhibit a good lifetime ( $>14$ days) in air. When the sensing of $300 \mathrm{ppb}$ ammonia takes only $30 \mathrm{~s}$ to deliver an apparent current drop, the recovery requires $300 \mathrm{~s}$ to let the current returns to its original value. When ammonia concentration increases to be $1 \mathrm{ppm}$, the recovery time is as long as $490 \mathrm{~s}$. Using blue light irradiation during the recovery period can greatly reduce the recovery time to be less than $60 \mathrm{~s}$. It was confirmed that such a photo-assisted recovery is not mainly due to the improved gas desorption under light irradiation, but due to the current compensation effect. The current drop after ammonia detection is compensated by the current increase under light irradiation. As a result, the fast multiple-time sensing gradually increases the amount of remaining ammonia residue inside the sensing film. Nevertheless, because of the low targeting detection range of $100 \mathrm{ppb}$ to $2000 \mathrm{ppb}$, a certain amount of reacting sites in PBDTTT-C-T can still react with the following ammonia molecules after the first sensing process. 
A new kinetic balance of gas desorption and absorption can be reached, enabling a reliable multiple testing performance. The proposed photo-assisted measurement takes only $90 \mathrm{~s}$ to complete a detecting cycle. We verified that, within one hour, 20-times testing to ammonia concentration ranged from 100 ppb to $2000 \mathrm{ppb}$ delivers a reliable calibration curve. Therefore, we conclude that the photo-assisted gas sensing is a practical method to solve the long recovery time in organic-based gas sensors. The proposed sensitive and stable ammonia sensor, together with the fast-operated photo-assisted system, is promising for developing real applications in industrial gas sampling or on-line fish freshness testing.

\section{Acknowledgment}

The authors would like to acknowledge the support by Ministry of Science and Technology, Taiwan (Project 106-2112-M-032-006 and 107-2112-M-032-006)

\section{Appendix A. Supporting information}

Supplementary data associated with this article can be found in the online version

\section{References}

[1] Y. Yang, G. Zhang, H. Luo, J. Yao, Z. Liu, D. Zhang, Highly Sensitive Thin-Film Field-Effect Transistor Sensor for Ammonia with the DPP-Bithiophene Conjugated Polymer Entailing Thermally Cleavable tert-Butoxy Groups in the Side Chains, ACS Appl. Mater. Interfaces, 8 (2016) 3635-3643.

[2] C.F. Lu, C.W. Shih, C.A. Chen, A. Chin, W.F. Su, Tuning the Morphology of Isoindigo DonorAcceptor Polymer Film for High Sensitivity Ammonia Sensor, Adv. Funct. Mater., 28 (2018) 1803145.

[3] F. Zhang, G. Qu, E. Mohammadi, J. Mei, Y. Diao, Solution-Processed Nanoporous Organic Semiconductor Thin Films: Toward Health and Environmental Monitoring of Volatile Markers, Adv. Funct. Mater., 27 (2017) 1701117.

[4] A. Lv, Y. Pan, L. Chi, Gas Sensors Based on Polymer Field-Effect Transistors, Sensors, 17 (2017) 213.

[5] L.-Y. Chang, M.-Y. Chuang, H.-W. Zan, H.-F. Meng, C.-J. Lu, P.-H. Yeh, J.-N. Chen, One-Minute Fish Freshness Evaluation by Testing the Volatile Amine Gas with an Ultrasensitive PorousElectrode-Capped Organic Gas Sensor System, ACS Sens., 2 (2017) 531-539.

[6] V.R. Heerthana, R. Preetha, Biosensors: a potential tool for quality assurance and food safety pertaining to biogenic amines/volatile amines formation in aquaculture systems/products, Reviews in Aquaculture, 0 (2018) 1-14.

[7] M.Y. Chuang, C.C. Chen, H.W. Zan, H.F. Meng, C.J. Lu, Organic Gas Sensor with an Improved Lifetime for Detecting Breath Ammonia in Hemodialysis Patients, ACS Sens., 2 (2017) 17881795. 
[8] T. Hibbard, K. Crowley, F. Kelly, F. Ward, J. Holian, A. Watson, A.J. Killard, Point of Care Monitoring of Hemodialysis Patients with a Breath Ammonia Measurement Device Based on Printed Polyaniline Nanoparticle Sensors, Anal. Chem., 85 (2013) 12158-12165.

[9] H. Ishida, T. Satou, K. Tsuji, N. Kawashima, H. Takemura, Y. Kosaki, S. Shiratori, T. Agishi, The breath ammonia measurement of the hemodialysis with a QCM-NH3 sensor, Bio-Med. Mater. Eng., 18 (2008) 99-106.

[10] D.J. Kearney, T. Hubbard, D. Putnam, Breath Ammonia Measurement in Helicobacter pylori Infection, Dig. Dis. Sci., 47 (2002) 2523-2530.

[11] S.H. Yu, J. Cho, K.M. Sim, J.U. Ha, D.S. Chung, Morphology-Driven High-Performance Polymer Transistor-based Ammonia Gas Sensor, ACS Appl. Mater. Interfaces, 8 (2016) 6570-6576.

[12] B. Peng, S. Huang, Z. Zhou, P.K.L. Chan, Solution-Processed Monolayer Organic Crystals for High-Performance Field-Effect Transistors and Ultrasensitive Gas Sensors, Adv. Funct. Mater., 27 (2017) 1700999.

[13] F. Ge, Z. Liu, S.B. Lee, X. Wang, G. Zhang, H. Lu, K. Cho, L. Qiu, Bar-Coated Ultrathin Semiconductors from Polymer Blend for One-Step Organic Field-Effect Transistors, ACS Appl. Mater. Interfaces, 10 (2018) 21510-21517.

[14] M.-Y. Chuang, H.-W. Zan, P. Yu, Y.-C. Lai, H.-F. Meng, Gas permeable silver nanowire electrode for realizing vertical type sensitive gas sensor, Org. Electron., 15 (2014) 2769-2774.

[15] M.-Y. Chuang, J.-N. Chen, H.-W. Zan, C.-J. Lu, H.-F. Meng, Modulated gas sensor based on vertical organic diode with blended channel for ppb-regime detection, Sens. Actuator B-Chem., 230 (2016) 223-230.

[16] M.-Z. Dai, Y.-L. Lin, H.-C. Lin, H.-W. Zan, K.-T. Chang, H.-F. Meng, J.-W. Liao, M.-J. Tsai, H. Cheng, Highly Sensitive Ammonia Sensor with Organic Vertical Nanojunctions for Noninvasive Detection of Hepatic Injury, Anal. Chem., 85 (2013) 3110-3117.

[17] L. Li, P. Gao, M. Baumgarten, K. Müllen, N. Lu, H. Fuchs, L. Chi, High Performance Field-Effect Ammonia Sensors Based on a Structured Ultrathin Organic Semiconductor Film, Adv. Mater., 25 (2013) 3419-3425.

[18] Q. Wang, S. Wu, F. Ge, G. Zhang, H. Lu, L. Qiu, Solution-Processed Microporous Semiconductor Films for High-Performance Chemical Sensors, Adv. Mater. Interfaces., 3 (2016) 1600518.

[19] C. Kumar, G. Rawat, H. Kumar, Y. Kumar, A. Kumar, R. Prakash, S. Jit, Electrical and Ammonia Gas Sensing Properties of PQT-12/CdSe Quantum Dots Composite-Based Organic Thin Film Transistors, IEEE Sens. J., 18 (2018) 6085-6091.

[20] B. Nketia-Yawson, A.R. Jung, Y. Noh, G.-S. Ryu, G.D. Tabi, K.-K. Lee, B. Kim, Y.-Y. Noh, Highly Sensitive Flexible NH3 Sensors Based on Printed Organic Transistors with Fluorinated Conjugated Polymers, ACS Appl. Mater. Interfaces, 9 (2017) 7322-7330.

[21] S. Han, X. Zhuang, W. Shi, X. Yang, L. Li, J. Yu, Poly(3-hexylthiophene)/polystyrene (P3HT/PS) blends based organic field-effect transistor ammonia gas sensor, Sens. Actuator B-Chem., 225 (2016) 10-15. 
[22] D. Khim, G.-S. Ryu, W.-T. Park, H. Kim, M. Lee, Y.-Y. Noh, Precisely Controlled Ultrathin Conjugated Polymer Films for Large Area Transparent Transistors and Highly Sensitive Chemical Sensors, Adv. Mater., 28 (2016) 2752-2759.

[23] H. Luo, C. Yu, Z. Liu, G. Zhang, H. Geng, Y. Yi, K. Broch, Y. Hu, A. Sadhanala, L. Jiang, P. Qi, Z. Cai, H. Sirringhaus, D. Zhang, Remarkable enhancement of charge carrier mobility of conjugated polymer field-effect transistors upon incorporating an ionic additive, Sci. Adv., 2 (2016) e1600076.

[24] R. Jaisutti, J. Kim, S.K. Park, Y.-H. Kim, Low-Temperature Photochemically Activated Amorphous Indium-Gallium-Zinc Oxide for Highly Stable Room-Temperature Gas Sensors, ACS Appl. Mater. Interfaces, 8 (2016) 20192-20199.

[25] X. Zhou, K.F. Niu, Z. Wang, L.Z. Huang, L.F. Chi, An ammonia detecting mechanism for organic transistors as revealed by their recovery processes, Nanoscale, 10 (2018) 8832-8839.

[26] S. Tiwari, A.K. Singh, L. Joshi, P. Chakrabarti, W. Takashima, K. Kaneto, R. Prakash, Poly-3hexylthiophene based organic field-effect transistor: Detection of low concentration of ammonia, Sens. Actuator B-Chem., 171 (2012) 962-968. 


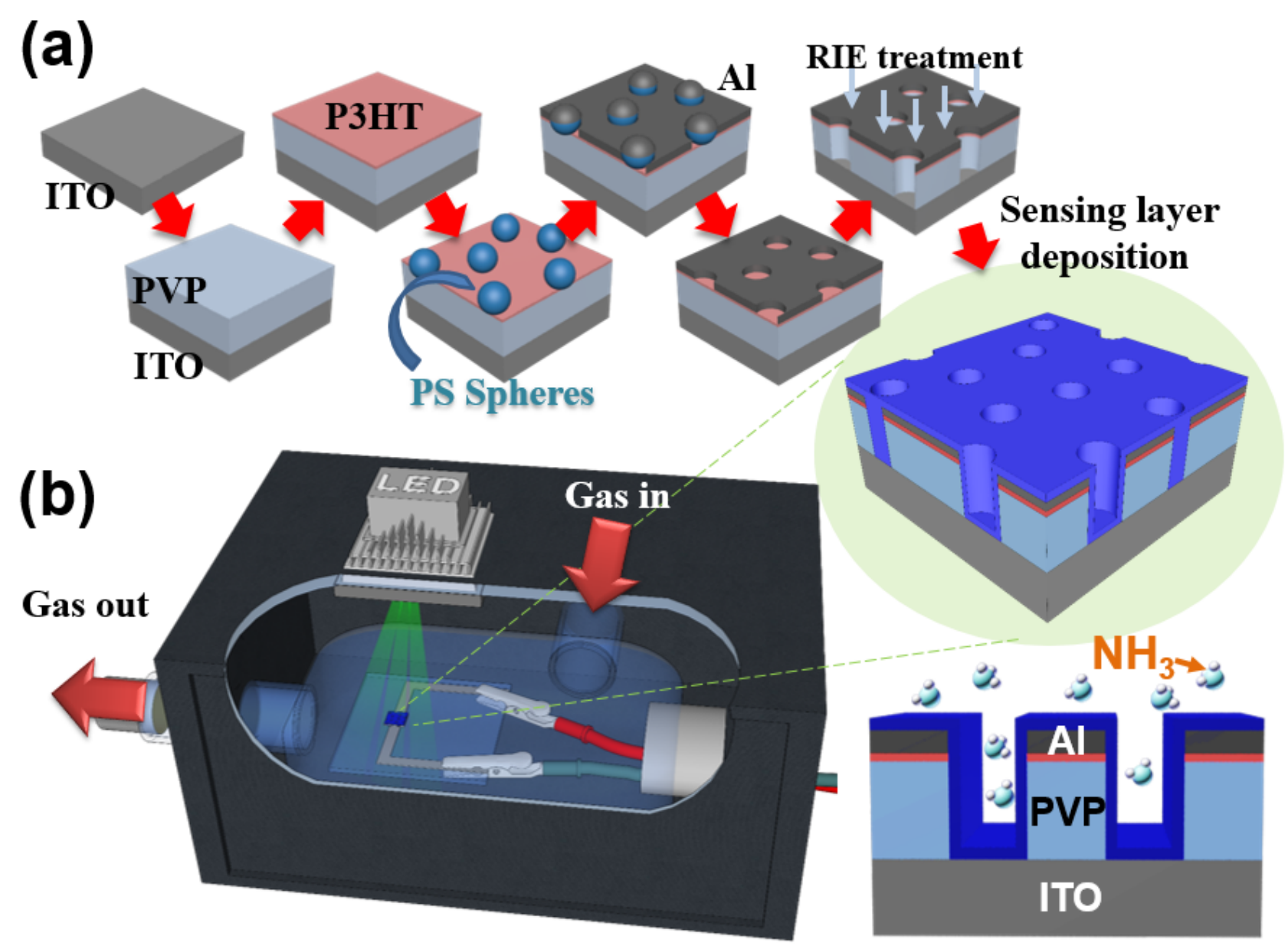

Fig. 1. Schematic diagram of (a) the fabrication process of cylindrical nanopores structure for organic ammonia sensor and (b) the design of light-assisted gas sensing system. 

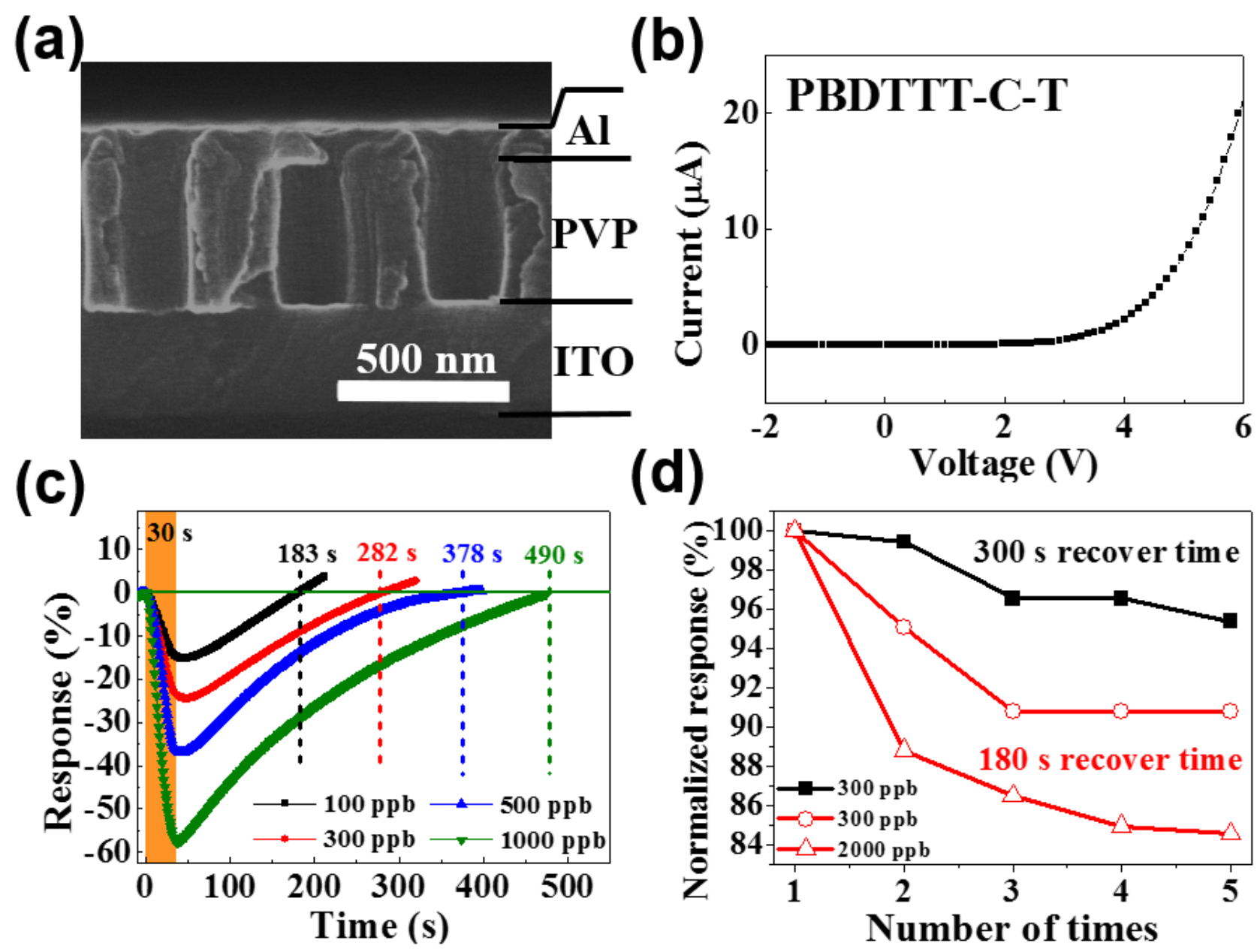

Fig. 2. (a) The scanning electron microscope (SEM) of the cylindrical nanopores structure. (b) The I$\mathrm{V}$ characteristic of PBDTTT-C-T sensor. (c) The real-time responses of PBDTTT-C-T sensor to ammonia concentration of $100 \mathrm{ppb}, 300 \mathrm{ppb}, 500 \mathrm{ppb}$, and $1000 \mathrm{ppb}$, respectively. The orange area indicates $30 \mathrm{~s}$ of the ammonia gas injection. The period for the response to recover to zero value was defined as the recovery time and is indicated at the respective sensing curve. (d) The normalized response of 5 times repeated sensing to $300 \mathrm{ppb}$, and $2000 \mathrm{ppb}$ ammonia concentration, respectively. The black and red curve indicated $300 \mathrm{~s}$ and $180 \mathrm{~s}$ recovery time between each sensing process, respectively. 


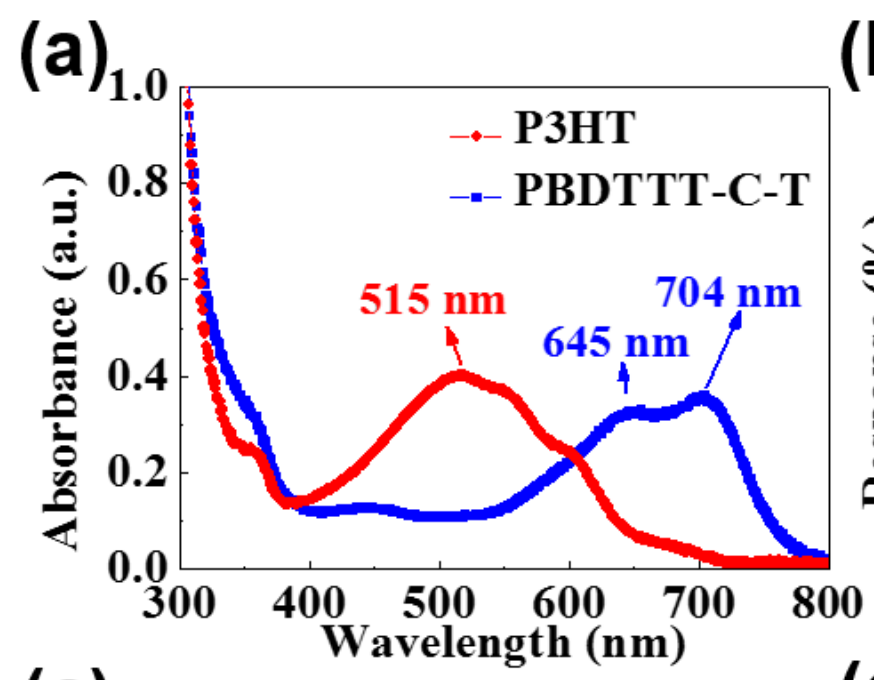

(b)
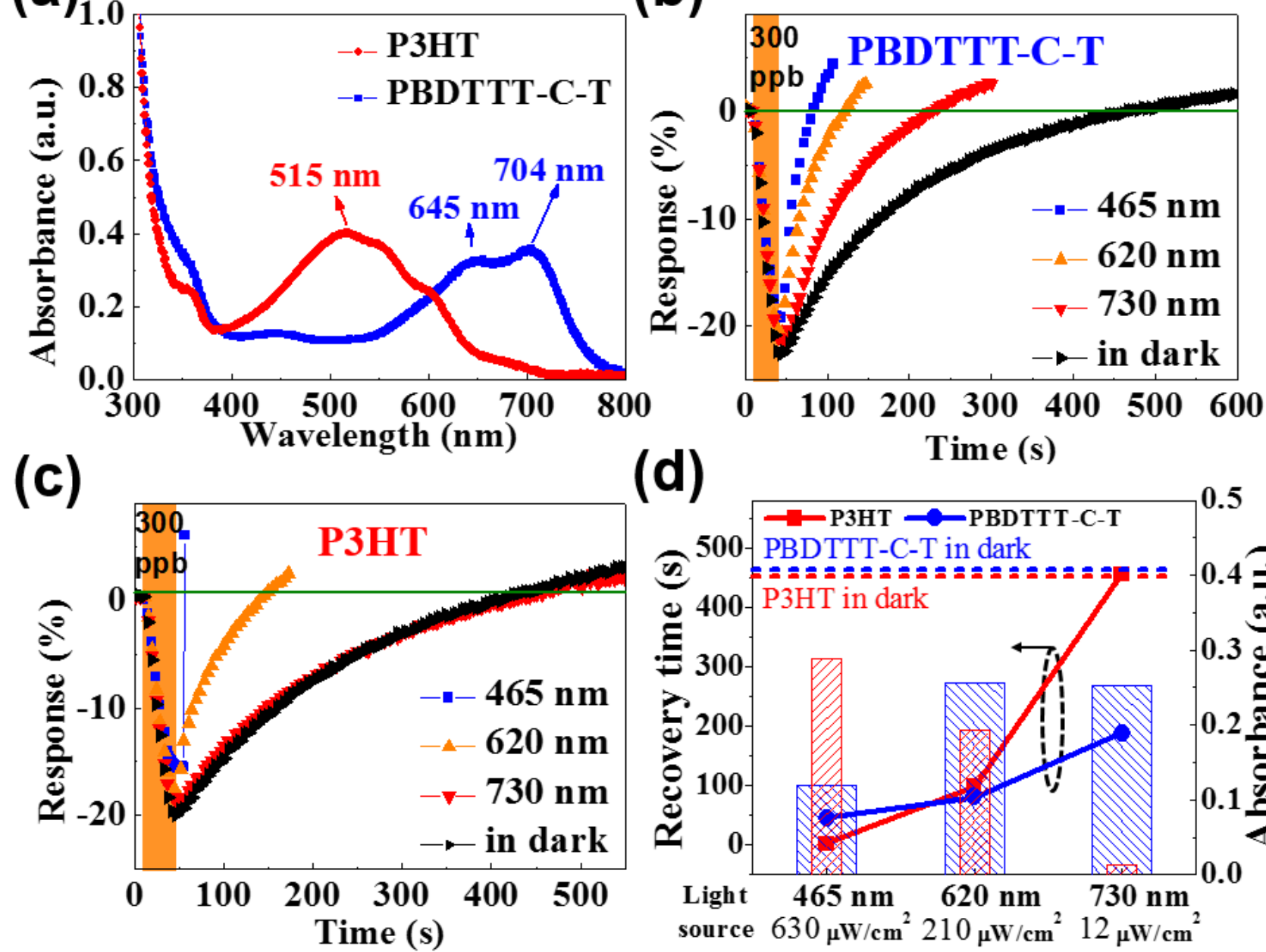

(d)

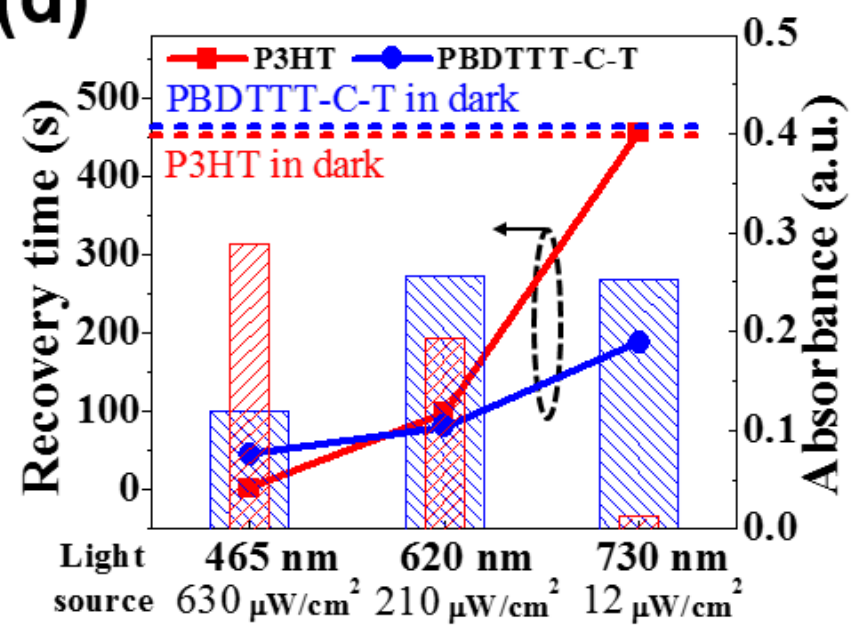

Fig. 3. (a) The UV-visible absorption spectrum of P3HT and PBDTTT-C-T organic polymer. The realtime measurement of (b) PBDTTT-C-T sensor and (c) P3HT sensor responses to $300 \mathrm{ppb}$ ammonia concentration in dark or under different light source irradiation. The wavelength of 3 LED light sources are 465, 620, and $730 \mathrm{~nm}$, respectively. (d) The dependency of recovery time and the corresponding absorbance of P3HT and PBDTTT-C-T sensor to the various wavelength light sources. The red and blue dot-lines indicate the recovery times without light irradiation for P3HT and PBDTTT-C-T sensors, respectively. 

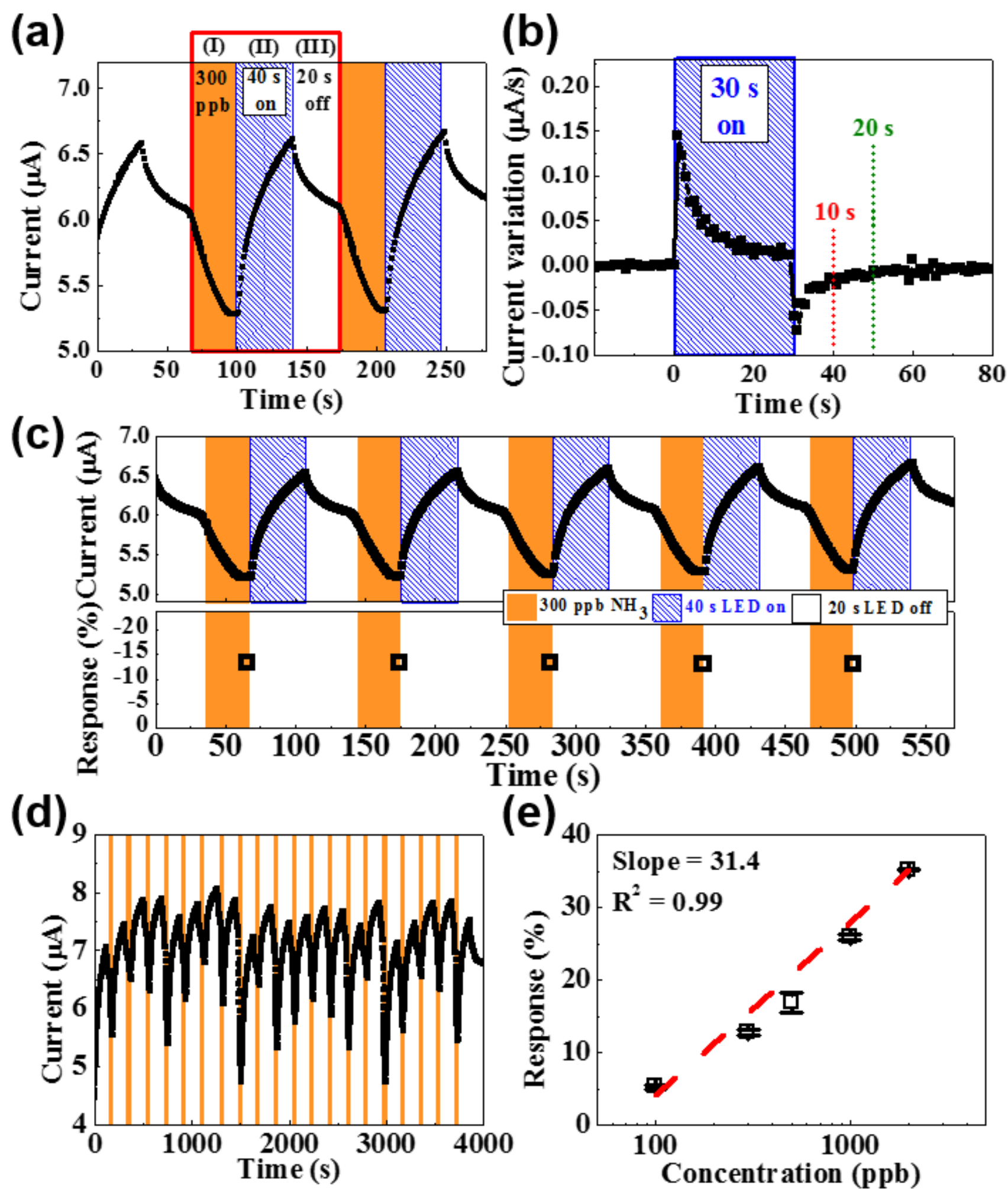

(e)

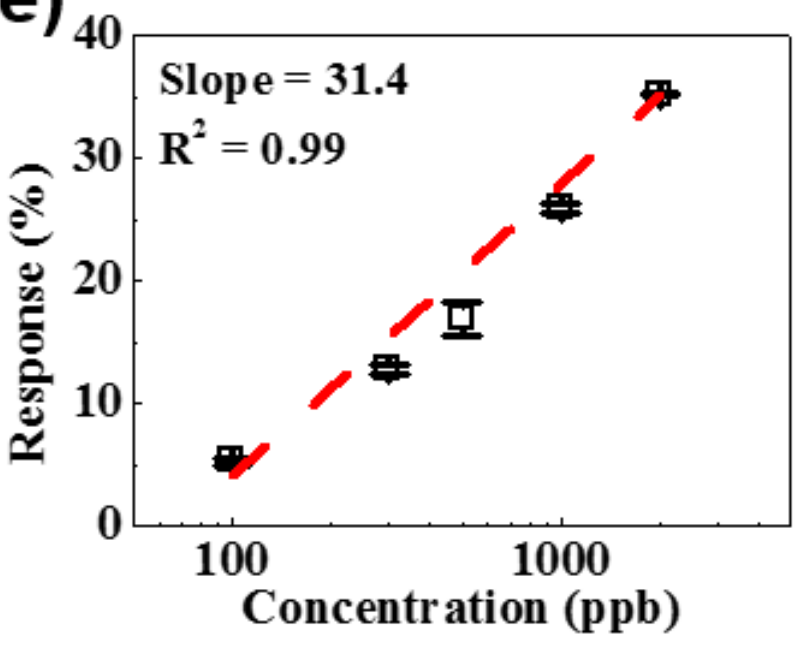

Fig. 4. (a) Real-time current measurement of PBDTTT-C-T sensor under three steps of the proposed light-assisted operation sequence: (I) Injection of ammonia for $30 \mathrm{~s}$. (sensing period, orange region). (II) LED irradiation for $40 \mathrm{~s}$. (recovery period, blue region). (III) Dark state for sensor stabilizing for 20 s. (stabilizing period, white region). (b) Current variation plotted as a function of time (c) Real-time current measurement and corresponding ammonia sensing response of PBDTTT-C-T sensor to 300 
ppb ammonia concentration for five repeated tests. (d) Real-time current measurement of PBDTTT-C$\mathrm{T}$ sensor responded to randomly injecting ammonia gas for 20-times. The introduced ammonia concentrations are ranging from $100 \mathrm{ppb}$ to $2000 \mathrm{ppb}$. (d) The extracted responses to different concentrations plotted as the sensing response as a function of ammonia concentration.

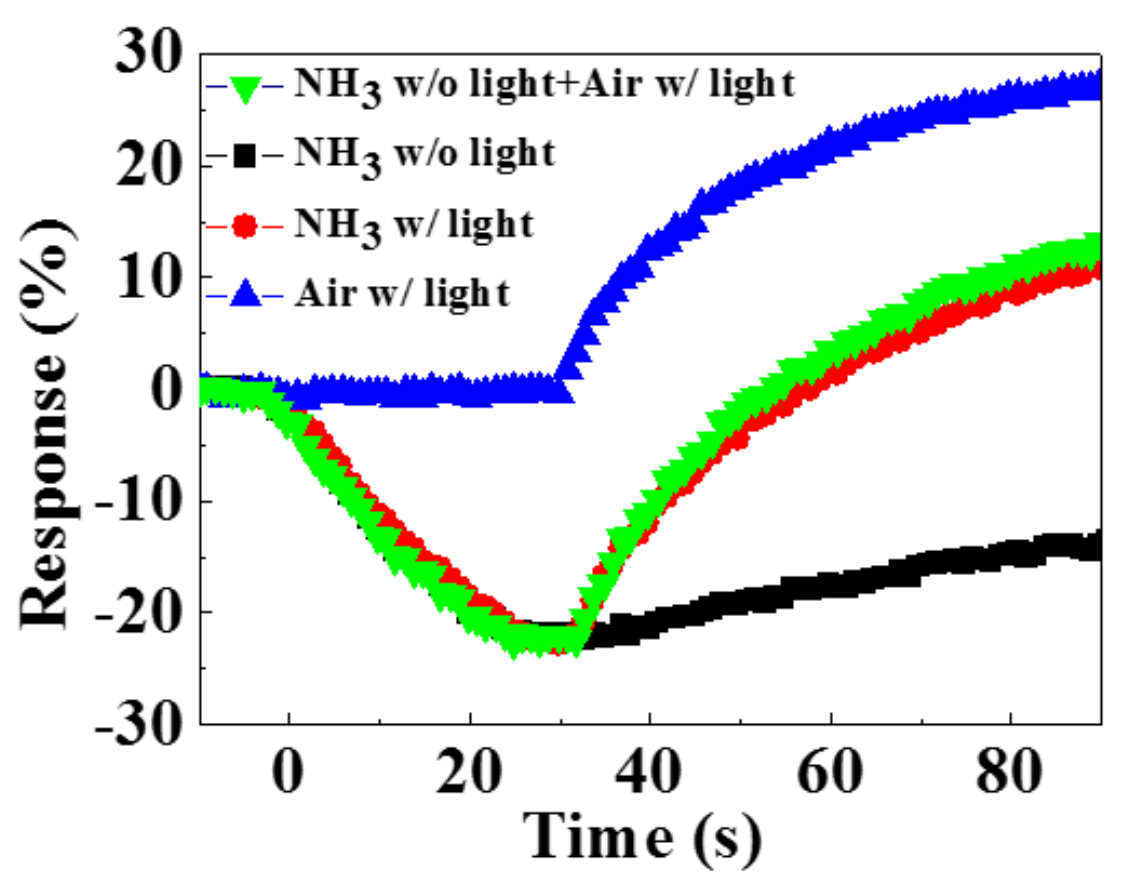

Fig. 5. Real-time responses of PBDTTT-C-T sensor in different conditions: The blue curve represents the condition of only light irradiation without gas exposure. The red curve represents the condition of the sensor firstly exposed to ammonia then recovered under light irradiation. The black curve represents the condition of only gas exposure without light irradiation. The green curve shows the superposition of blue and black curves. 

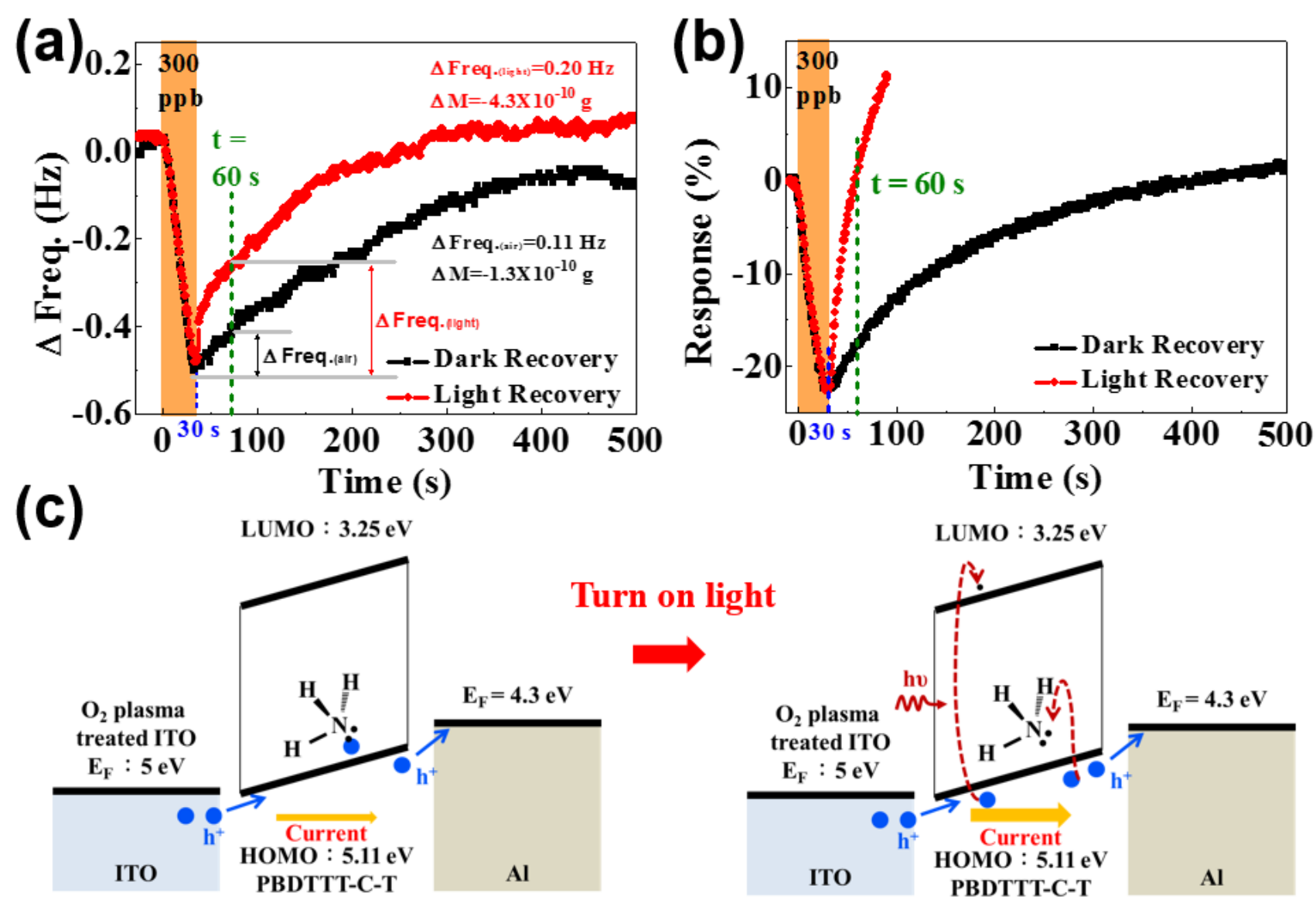

Fig. 6. (a) Real-time quartz crystal microbalance (QCM) measurement of PBDTTT-C-T thin film exposed to $300 \mathrm{ppb}$ ammonia then recovered with and without light irradiation. (b) Real-time response of PBDTTT-C-T sensor exposed to $300 \mathrm{ppb}$ ammonia then recovered with and without light irradiation. (c) The schematic drawing of the ammonia sensing mechanism in PBDTTT-C-T sensor and the mechanism of the proposed light-assisted recovery. 


\section{Supporting information}

\section{Photo-Assisted Recovery in Ammonia Sensor Based on Organic Vertical Diode}

Yu-Ting Lin ${ }^{\mathrm{a}}$, Shang-Yu Yu ${ }^{\mathrm{b}, \mathrm{c}}$, Hsiao-Wen Zan ${ }^{\mathrm{b} *}$, Ping-Hung Yeh ${ }^{\mathrm{d}}$, Chia-Jung Lu ${ }^{\mathrm{e}}$, Hsin-Fei Meng ${ }^{\mathrm{f}}$, Chih-Wei Luo ${ }^{\mathrm{a}}$, and Olivier Soppera ${ }^{\mathrm{c}}$

${ }^{a}$ Department of Electrophysics, ${ }^{b}$ Department of Photonics , College of Electrical and Computer Engineering, and ${ }^{\mathrm{f}}$ Institute of Physics, National Chiao Tung University, No.1001, University Rd., East Dist., Hsinchu City 300, Taiwan

${ }^{c}$ Université de Haute-Alsace, CNRS, IS2M UMR 7361, F-68100 Mulhouse, Université de Strasbourg, France

${ }^{\mathrm{d}}$ Department of Physics, Tamkang University, No.151, Yingzhuan Rd., Tamsui Dist., New Taipei City 25137, Taiwan

${ }^{\mathrm{e}}$ Department of Chemistry, National Taiwan Normal University, No.162, Heping East Rd., Section 1, Taipei City 106, Taiwan

*Corresponding authors

E-mail :

hsiaowen@mail.nctu.edu.tw (Hsiao-Wen Zan)

phyeh331@mail.tku.edu.tw (Ping-Hung Yeh)

ABSTRACT: The supporting information includes the schematic drawing of the designed light-assisted gas sensing system and the decayed responses of PBDTTT-C-T sensor and P3HT sensor on different days. 


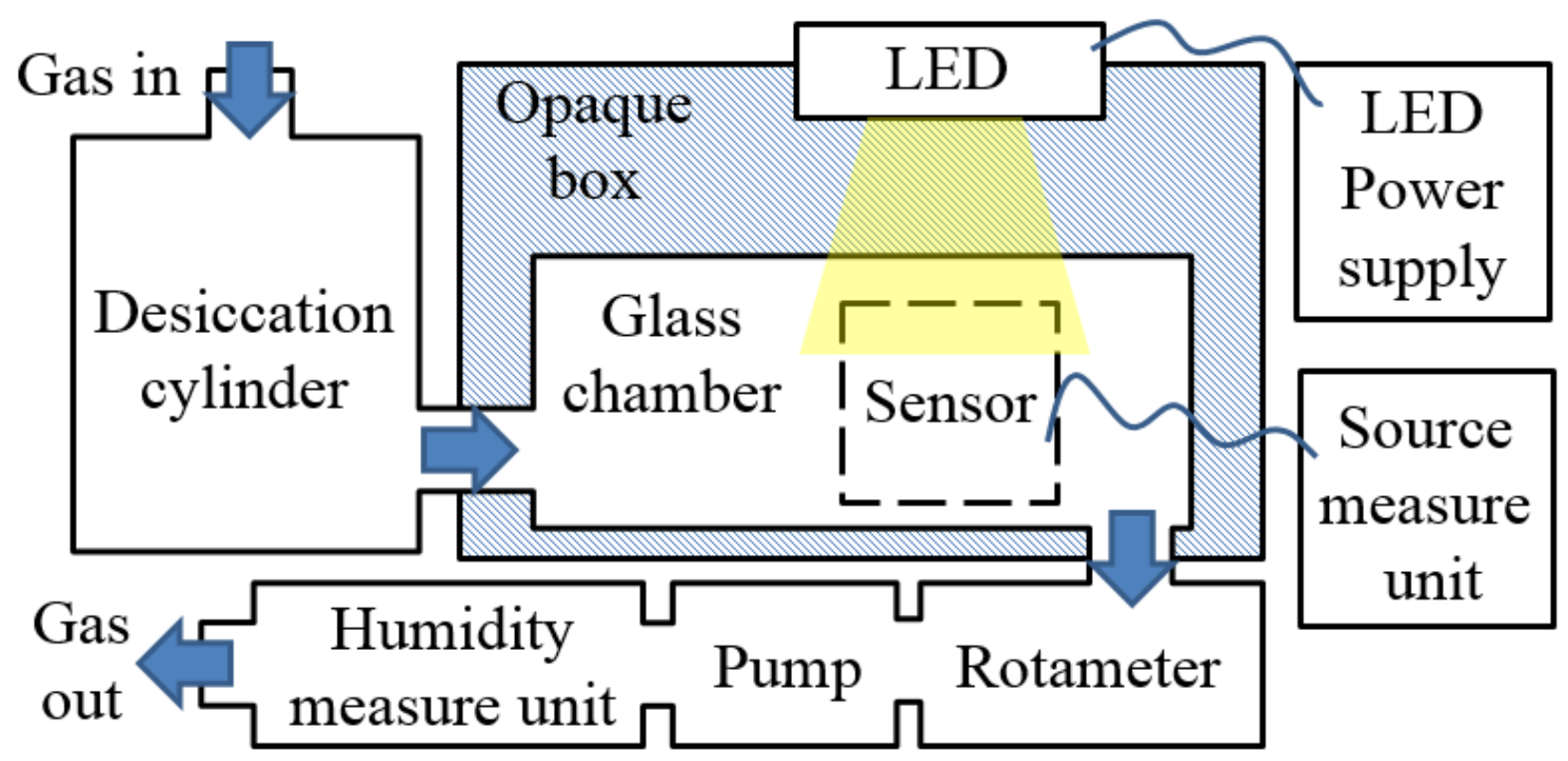

Fig. S1. The schematic drawing of the designed light-assisted gas sensing system. The blue arrows indicated the gas flow direction.

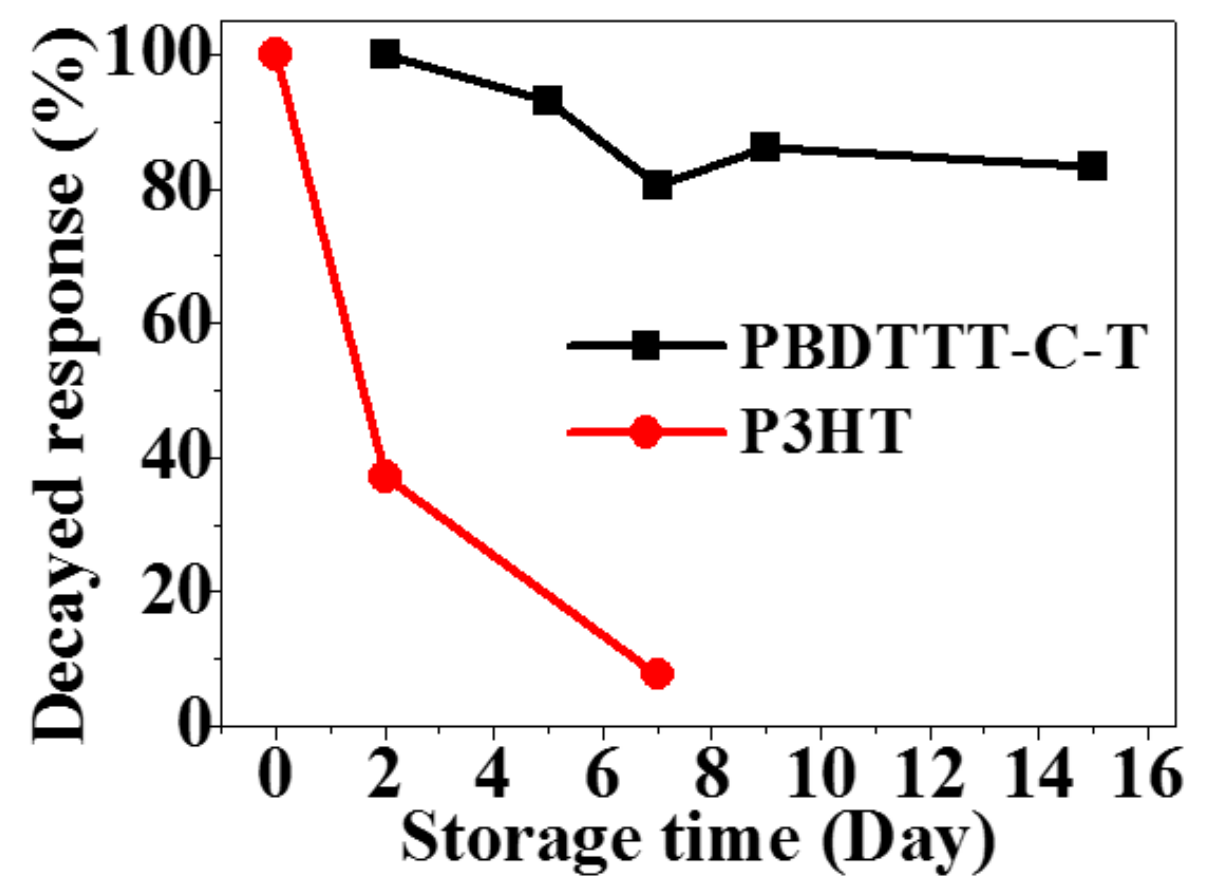

Fig. S2. The decayed responses of PBDTTT-C-T and P3HT sensors in different days. The decayed response is defined as the sensing response divided by its initial response value. 